\title{
Acupuncture and Electracupuncture Therapy- A Part of Complementary Medicine - Review of The Literature
}

\author{
Mihaela Mandu ${ }^{1 *}$ and Cosmin Daniel Oprea ${ }^{2}$ \\ ${ }^{1}$ Bagdasar Arseni Clinical Emergency Hospital, Bucharest, Romania
}

${ }^{2}$ Filantropia Municipal Hospital, Craiova, Romania

*Corresponding author: Mihaela Mandu, Bagdasar Arseni Clinical Emergency Hospital, Bucharest, Romania

\begin{abstract}
Although the benefits of acupuncture have been known for thousands of years, the mechanisms by which it acts are not fully elucidated. The present paper aims to bring the benefits of electroacupuncture as alternative medicine, making a literature review. The positive effects of acupuncture and electroacupuncture in medical conditions such as chronic nonspecific low back pain, depression, anxiety, drug dependence, alcohol, schizophrenia, Alzheimer's disease, rheumatic diseases, neurological diseases, cardiovascular disorders, infertility, chemotherapy. According to some authors, electroacupuncture is superior to classical acupuncture, while others support the need for further studies to clarify this dilemma. The fact is that the clinical benefits of both acupuncture and electroacupuncture are unquestionable.
\end{abstract}

Keywords: Electroacupuncture; Acupuncture; Therapy; Alternative; Medicine

\section{Introduction}

Although the benefits of acupuncture have been known for thousands of years, the mechanisms by which it acts are not fully elucidated [1]. It is based on the energy meridian theory and originates in China, with a great spread in Europe [2]. The term electroacupuncture was invented by the French acupuncturist Dr Roger de La Fuye around 1947 [3,4]. It linked acupuncture stimuli to an electrical device with remarkable clinical results $[3,4]$.

\section{Material and Method}

The present paper aims to bring the benefits of electroacupuncture as alternative medicine, making a literature review. There are numerous studies in the specialized literature that highlight the beneficial effects of electroacupuncture on various human pathologies, especially chronic pain. Chronic Nonspecific Low Back Pain (CNLBP) is one of the most common diseases that cause disability in the world. A double-blinded and placebo-controlled randomized clinical trial conducted in 2018 highlighted the fact that 10 electroacupuncture sessions reduced pain intensity both at rest and on movement [5]. There are also several studies that demonstrate the definite benefits of acupuncture in CNLBP compared with placebo groups [6]. It seems that electroacupuncture would be more effective compared to classical acupuncture [7]. At the same time, a growing number of studies on animals highlight the benefits of acupuncture in reducing inflammation, which is particularly useful in rheumatologic pathologies such as rheumatoid arthritis [8]. Also, both acupuncture and electroacupuncture have clinical benefits in the treatment of psychiatric problems such as depression, anxiety, drug addiction, alcohol $[7,9]$. There was an increase in the level of ACTH, beta endorphins, serotonin and noradrenaline in patients with schizophrenia, the severity of the disease being diminished [9]. Also, acupuncture increases the level of melatonin in the evening, which is beneficial in insomnia [10]. A literature review published in 2019 in Frontiers in psychiatry has highlighted the positive effects of acupuncture on neuropsychiatric illness (depression, anxiety, schizophrenia and Alzheimer's disease), yet further studies are needed to fully understand the pathophysiological mechanisms by which this technique derived from Traditional Chinese Medicine acts in these neuropsychiatric diseases and to establish protocols for treatment guidelines [11]. At the same time, the effects of 
acupuncture on the nervous system and cramps have been studied since 1972, detecting 3 phases of acupuncture: "on the $1^{\text {st }}$ phaseVaso-constriction nervous system; $2^{\text {nd }}$ phase-Quasi-control; $3^{\text {rd }}$ phase-Vaso-dilation" [12]. Another prospective randomized trial showed improved pregnancy rate after assisted reproduction therapy (ART) in patients who followed acupuncture versus the group of patients who did not follow ART with acupuncture (the documented clinical pregnancies had a $42.5 \%$ incidence in the group with acupuncture versus $26.3 \%$ in the acupuncture group) [13]. Acupuncture appears to be beneficial to patients who are undergoing chemotherapy and have side effects such as nausea and vomiting [14]. Another application of electroacupuncture is in medical recovery in patients with hemiplegia following strokes. It was found that patients treated with recovery techniques along with electroacupuncture had a shorter duration of hospitalization than the other group that was treated only by recovery techniques. Also, neurological and functional outcomes were clearly superior, increasing patient quality and independence [15].

\section{Results}

Data from the literature is quite broad but not enough to fully understand the mechanisms by which acupuncture and electroacupuncture act in different pathologies. However, the clinical effects are clear. It seems that electroacupuncture is superior to classical acupuncture after some authors [7]. The remarkable effects of these alternative Chinese medicine therapies are reflected in psychiatric disorders such as depression, insomnia, alcohol addiction, drugs, schizophrenia, Alzheimer's disease [7,9-11]. They also produce analgesia and hinder inflammation. There are studies that prove the effectiveness of acupuncture in infertility problems [13]. There are studies that demonstrate that acupuncture reduces side effects of chemotherapy in oncology patients [14]. The combination of methods of medical recovery and electroacupuncture is beneficial in patients with hemiplegia after stroke [15].

\section{Conclusion}

Both acupuncture and electroacupuncture are two procedures with definite clinical benefits in various human pathologies such as chronic nonspecific low back pain, depression, anxiety, drug addiction, alcohol, schizophrenia, Alzheimer's disease, rheumatic diseases, neurological diseases, cardiovascular diseases, infertility, side effects of chemotherapy. However, research in this area is increasingly mercurial trying to find the mechanism by which these alternative medicine techniques work in the human body.

\section{References}

1. Ji-Yeun Park, Uk Namgung (2018) Electroacupuncture therapy in inflammation regulation: current perspectives. J Inflamm Res 11: 227237.

2. A White, E Ernst (2004) A brief history of acupuncture. Rheumatology 43(5): 662-663.

3. http://www.biopathica.co.uk/documents/eav/voll.pdf

4. https://www.amcollege.edu/blog/what-is-electroacupuncture-amcmiami

5. Paula M S Leite, Andreza R C Mendonça, Leonardo Y S Maciel, Maurício L Poderoso Neto, Carla C A Araujo, et al. (2018) Does Electroacupuncture Treatment Reduce Pain and Change Quantitative Sensory Testing Responses in Patients with Chronic Nonspecific Low Back Pain? A Randomized Controlled Clinical Trial. Evid Based Complement Alternat Med, pp. 8586746.

6. Comachio J, Oliveira Magalhães $M$, Nogueira Burke T, Vidal Ramos LA, Peixoto Leão Almeida G, et al. (2015) Efficacy of acupuncture and electroacupuncture in patients with nonspecific low back pain: study protocol for a randomized controlled trial. Trials 16: 469.

7. George A Uletta, Songping Hanb, Ji sheng Hanc (1998) Electroacupuncture: mechanisms and clinical application. Biological Psychiatry 44 (2): 129-138.

8. Ji Yeun Park, Uk Namgung (2018) Electroacupuncture therapy in inflammation regulation: current perspectives. J Inflamm Res 11: 227 237.

9. Noah Samuels, Cornelius Gropp, Shepherd Roee Singer, Menachem Oberbaum (2008) Acupuncture for Psychiatric Illness: A Literature Review. Behavioral Medicine, 34(2): 55-64.

10. D Warren Spence, Leonid Kayumov, Adam Chen, Alan Lowe, Umesh Jain, et al. (2004) Acupuncture Increases Nocturnal Melatonin Secretion and Reduces Insomnia and Anxiety: A Preliminary Report. The Journal of Neuropsychiatry and Clinical Neurosciences 16(1): 19-28.

11. Tu Cheng Hao, MacDonald Iona, Chen Yi-Hung (2019) The Effects of Acupuncture on Glutamatergic Neurotransmission in Depression, Anxiety, Schizophrenia, and Alzheimer's Disease: A Review of the Literature. Front Psychiatry.

12. Omura, Yoshiaki (1975) Pathophysiology of acupuncture treatment: effects of acupuncture on cardiovascular and nervous systems. Acupuncture \& Electro-Therapeutics Research 1(1-4): 51-141.

13. Wolfgang Epaulus, Mingmin Zhang, Erwin Strehler, Imam El-Danasouri, Karl Sterzik (2002) Influence of acupuncture on the pregnancy rate in patients who undergo assisted reproduction therapy. Fertility and Sterility 77(4): 721-724.

14. Jeanette Ezzo, Andrew Vickers, Mary Ann Richardson, Claire Allen, Suzanne L Dibble et al. (2005) Acupuncture-Point Stimulation for Chemotherapy-Induced Nausea and Vomiting. Journal of Clinical Oncology 23(28): 7188-7198.

15. Wong Alice M K, Su Ting Ya, Tang Fuk Tan, Cheng Pao Tsai, Liaw Mei Yun (1999) Clinical trial of electrical acupuncture on hemiplegic stroke patients. American Journal of Physical Medicine \& Rehabilitation 78(2): $117-122$. 
(C)

To Submit Your Article Click Here: Submit Article

DOI: $10.32474 /$ OAJCAM.2019.01.000122

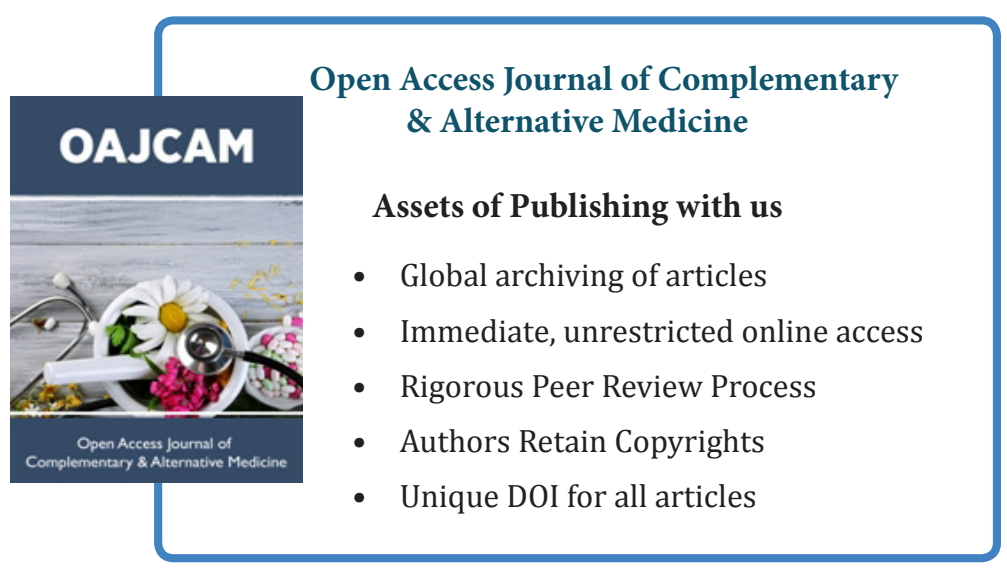

